\title{
Impacto del protocolo propuesto por la American Society for Gastrointestinal Endoscopy en pacientes de alto riesgo de coledocolitiasis en el Hospital Regional ISSSTE Puebla en México
}

\author{
Impact of the protocol proposed by the American Society for Gastrointestinal Endoscopy in
} patients at high risk of choledocholithiasis, in Puebla Regional Hospital ISSSTE in Mexico

\section{Alberto De Jesús-Flores* y Gustavo A. Guerrero-Martínez}

Servicio de Cirugía General, Hospital Regional de Alta Especialidad, Instituto de Seguridad y Servicios Sociales de los Trabajadores del Estado, Puebla, Puebla, México

\section{Resumen}

Antecedentes: La coledocolitiasis es una de las principales complicaciones de la colelitiasis, con una prevalencia en la población mexicana del 14\%. El manejo a través de la guía propuesta por la American Society for Gastrointestinal Endoscopy (ASGE) en 2010 puede apoyar al cirujano para la resolución oportuna de la patología. Objetivo: Determinar que el uso de la guía de la ASGE para el manejo de coledocolitiasis en pacientes con "alto riesgo" reduce las complicaciones de esta patología. Método: Estudio comparativo, retrospectivo, de casos y controles, del 1 de enero de 2015 al 31 de diciembre de 2017. Se incluyeron pacientes que se estratificaron en alto riesgo de coledocolitiasis de acuerdo con la guía ASGE y se dividieron en dos grupos: el grupo de casos (diagnóstico por guía ASGE) fue sometido directamente a colangiopancreatografía retrógrada endoscópica (CPRE), y el otro grupo (no ASGE) siguió el protocolo de realizar tomografía computarizada, colangiorresonancia magnética y finalmente CPRE. Resultados: De 61 pacientes con coledocolitiasis, se comparó entre los grupos la aparición de colangitis, las complicaciones posteriores a la CPRE, el tiempo de realización de la CPRE, los días de estancia hospitalaria y la mortalidad. Se obtuvo diferencia significativa en complicaciones tras la CPRE (grupo ASGE 3/35 [9\%], grupo no ASGE 8/26 [30.7\%]; odds ratio [OR]: 0.2; intervalo de confianza del 95\% [IC 95\%]: 0.050.9; $p=0.03$ ) y en colangitis (grupo ASGE 2/35 [5.7\%], grupo no ASGE 8/26 [30-7\%]; OR: 0.13; IC 95\%: 0.03-0.71; $p=$ 0.009). Conclusión: En nuestro medio, el empleo de la guía ASGE reduce la presencia de colangitis y las complicaciones tras la CPRE.

PALABRAS CLAVE: ASGE. Coledocolitiasis. CPRE.

\begin{abstract}
Background: Choledocholithiasis is one of the main complications of cholelithiasis with a prevalence in the Mexican population of 14\%. The management through the guide proposed by the American Society for Gastrointestinal Endoscopy (ASGE) in 2010 can support the surgeon for the timely resolution of the pathology. Objective: To determine that the guide proposed by ASGE for the management of choledocholithiasis in patients with "high risk" reduces the complications of this pathology. Methods: A comparative, retrospective case-control study was conducted from January 1, 2015 to December 31, 2017. We included patients who were stratified at high risk of choledocholithiasis according to the ASGE guidelines. They were divided into 2
\end{abstract}

\author{
Correspondencia: \\ *Alberto De Jesús-Flores \\ Circuito Juan Pablo II, 2417 \\ Col. La Hacienda \\ C.P. 72570 , Puebla, Pue., México \\ E-mail: betoelmagodeoz@gmail.com
}

Fecha de recepción: 24-08-2018

Fecha de aceptación: 26-02-2019

DOI: $10.24875 / C I R U .19000683$
Cir Cir. 2019;87:423-427

Contents available at PubMed www.cirugiaycirujanos.com 
groups: the case group (diagnosis by ASGE guidelines) was submitted directly to endoscopic retrograde cholangiopancreatography (ERCP), the second group (no ASGE) followed the protocol of performing computed tomography, magnetic cholangioresonance, and finally ERCP. Results: Of 61 patients with choledocholithiasis, the following groups were compared: the appearance of cholangitis, the complications after ERCP, time of ERCP, days of hospital stay and mortality. Obtaining significant difference in: "complications post ERCP" group ASGE 3/35 (9\%), group no ASGE 8/26 (30.7\%) with OR of 0.2 (0.05 - 0.9 IC 95\%) and a $p=0.03$; "Cholangitis" group ASGE 2/35 (5.7\%), group no ASGE 8/26 (30-7\%) with ORP 0.13 (0.03-0.71 IC $95 \%)$ and $p=0.009$. Conclusion: In our setting, the use of the ASGE guideline reduces the presence of cholangitis and post-ERCP complications.

KEY WORDS: ASGE. Choledocholithiasis. Endoscopic retrograde cholangiopancreatography.

\section{Introducción}

La coledocolitiasis se define como la presencia de cálculos biliares en el conducto biliar común. Colombo describió de forma inicial la coledocolitiasis al realizar la necropsia de san Ignacio de Loyola; con posterioridad, Thornton efectuó en 1889 la primera exploración exitosa de las vías biliares al extraer a través de una coledocotomía 188 cálculos a su paciente ${ }^{1}$.

La prevalencia de coledocolitiasis en pacientes con colelitiasis es cercana al $15 \%$, y hasta el $10 \%$ de los sometidos a colecistectomía laparoscópica padecerán de litos en la vía biliar común. El diagnóstico de coledocolitiasis requiere un alto grado de sospecha, y para su manejo se utilizan diversos estudios de imagen con elevado costo, como tomografía, resonancia magnética y colangiografía retrógrada endoscópica (CPRE)².

La mayoría de los pacientes presentan ictericia, acolia, dolor abdominal y en ocasiones fiebre, que se acompañan de pruebas alteradas de la función hepática (elevación de bilirrubina y fosfatasa alcalina). Los pacientes con coledocolitiasis no complicada pueden incluso permanecer asintomáticos, y el diagnóstico se basa en la sospecha por las alteraciones de la función hepática. Un paciente se considera complicado cuando la coledocolitiasis se acompaña de colangitis o pancreatitis biliar ${ }^{3}$.

Los estudios de imagen tales como ultrasonido, resonancia magnética y CPRE son de gran apoyo 4 .

En 2010, la American Society for Gastrointestinal Endoscopy (ASGE) presento una guía para evaluar el riesgo de presentar coledocolitiasis de acuerdo con los siguientes parámetros:

- Predictores muy fuertes:

- Bilirrubina $>4 \mathrm{mg} / \mathrm{dl}$.

- Datos clínicos de colangitis ascendente.

- Lito en la vía biliar visto por ultrasonido.
- Predictores fuertes:

- Vía biliar extrahepática dilatada (> $6 \mathrm{~mm}$ ).

- Bilirrubina total 1.8-4.0 mg/dl.

- Predictores moderados:

- Alteración de la función hepática.

- Edad > 55 años.

- Cuadro clínico de pancreatitis biliar.

De acuerdo con los factores predictivos anteriores, los pacientes se clasifican de la siguiente forma:

- Alto riesgo:

- Un predictor muy fuerte.

- Dos predictores fuertes.

- Riesgo intermedio:

- Un predictor fuerte o al menos uno moderado.

- Riesgo bajo:

- Sin factores predictivos.

La bilirrubina total $>1.7 \mathrm{mg} / \mathrm{dl}$ tiene una especificidad del $60 \%$ para coledocolitiasis, y aumenta al $75 \%$ con un valor $>4 \mathrm{mg} / \mathrm{dl}$. El ultrasonido tiene una baja sensibilidad para el diagnóstico de litos en la vía biliar (22-55\%), pero aumenta para la detección de dilatación de la vía biliar (77-87\%), un hallazgo asociado a coledocolitiasis. La tomografía helicoidal tiene una sensibilidad del $65-88 \%$ y una especificidad del $73-97 \%$ para coledocolitiasis; el costo y la exposición a radiación (similar a 500 radiografías simples) limitan su uso. La resonancia magnética presenta una sensibilidad del $87-92 \%$ y una especificidad del $93-97 \% \%^{4-7}$.

La CPRE, con alta capacidad resolutiva, cuyo éxito en la extracción del lito se encuentra en el $74.4-100 \%$, presenta una morbilidad del $5 \%$. También cuenta con sus complicaciones al ser cruenta, como pancreatitis (1.3-6.7\%), infección (0.6-5\%), sangrado (0.3-2.0\%) y perforación $(0.1-1.1 \%)^{4,8}$.

El principio básico del tratamiento de la coledocolitiasis es la extracción del cálculo de la vía biliar, lo cual puede realizarse por vía endoscópica o quirúrgica.

La ASGE recomienda una CPRE preoperatoria en los pacientes con alto riesgo de padecer coledocolitiasis de 
acuerdo a su algoritmo para el diagnóstico y el manejo de pacientes con probable coledocolitiasis².

La prevalencia de esta patología en el Hospital Regional ISSSTE Puebla, Puebla, es similar a lo reportado en otras partes del mundo. El objetivo de este estudio fue determinar si el uso del protocolo ASGE disminuye la morbilidad (colangitis), la mortalidad, los días de estancia hospitalaria y los días de resolución en los pacientes con alto riesgo de coledocolitiasis.

\section{Método}

Estudio comparativo, observacional, transversal y ambilectivo, del 1 de enero de 2015 al 31 de diciembre de 2017.

\section{Población de estudio}

Mediante muestreo no probabilístico se seleccionaron los pacientes que presentaron alto riesgo de presentar coledocolitiasis.

\section{Definición del grupo de casos}

El primer grupo (ASGE) cumplió los factores predictivos (clínicos, bioquímicos, ultrasonido) para ser de alto riesgo y se les realizó primariamente CPRE.

\section{Definición del grupo control}

El segundo grupo (método tradicional) siguió el protocolo de realizar pruebas de laboratorio, ultrasonido, tomografía abdominal simple o contrastada, y colangiorresonancia magnética, para constatar el diagnóstico de coledocolitiasis; en caso de fuerte sospecha se realizó CPRE.

\section{Criterios de inclusión}

Pacientes mayores de edad sin colecistectomía previa, que presentaran criterios de alto riesgo de coledocolitiasis de acuerdo con la guía ASGE (un valor predictivo muy fuerte o dos valores predictivos fuertes), que contaran con expediente clínico completo y notas médicas en las que se especificaran las herramientas diagnósticas utilizadas para la detección de la coledocolitiasis.

\section{Criterios de exclusión}

Pacientes menores de edad, que no contaran con expediente clínico completo o fuera inexistente, o que a su ingreso ya presentaran alguna complicación de la coledocolitiasis (colangitis, pancreatitis biliar aguda).

\section{Criterios de eliminación}

Pacientes en quienes se protocolizó otra enfermedad, aunque tuviera alto riesgo de coledocolitiasis.

\section{Resultados}

Se obtuvo una muestra de 66 pacientes con alto riesgo para coledocolitiasis. Se comparó el porcentaje de diagnóstico definitivo de coledocolitiasis.

\section{Diagnóstico de coledocolitiasis}

En el grupo ASGE se descartó la coledocolitiasis en $3 / 38$ pacientes $(7.8 \%$ ), y en el grupo no ASGE se descartó con colangiorresonancia en 2/28 pacientes (7.1\%). De los 61 pacientes con coledocolitiasis, $48(78.7 \%)$ eran mujeres y $13(21.3 \%)$ eran hombres. El rango de edad fue de 18-93 años, con un promedio de 46 años.

El grupo ASGE estuvo conformado por 35 (57.4\%) pacientes y el grupo no ASGE por 26 (42.6\%). Los días de estancia hospitalaria fueron $7.2 \pm 2.9$ en el grupo ASGE y $7.7 \pm 2.9$ en el grupo no ASGE. Los días de espera para la realización de CPRE fueron $2.6 \pm 1.2$ para el grupo ASGE y $5.4 \pm 1.9$ para el grupo no ASGE. Durante su estancia hospitalaria, 10 (16.4\%) pacientes se complicaron con colangitis, de los cuales dos fueron del grupo ASGE y ocho del grupo no ASGE. Una paciente (1.6\%) del grupo no ASGE, de 73 años, falleció por colangitis durante la realización del estudio. Del total de los pacientes, 11 (18\%) de ellos tuvieron alguna complicación posterior a la CPRE.

\section{Colangitis}

Hubo una mayor frecuencia de colangitis en el grupo de diagnóstico por el método tradicional (8/30; $7 \%$ ), con una odds ratio (OR) de 0.13 (protectora en caso de diagnóstico a través de guía ASGE) calculada con la prueba de ji al cuadrado $(p=0.009)$.

\section{Complicaciones tras la CPRE}

Hubo una mayor frecuencia de complicaciones en el grupo de diagnóstico por el método tradicional 
Tabla 1. Asociación de la frecuencia de complicaciones en pacientes con coledocolitiasis diagnósticados a través de la guía ASGE de 2015 a 2017

\begin{tabular}{lcccr}
\hline Variable & $\begin{array}{c}\text { Diagnostico ASGE } \\
(\mathbf{n}=\mathbf{3 5}) \text { Fcia (\%) }\end{array}$ & $\begin{array}{c}\text { Diagnostico convencional } \\
(\mathbf{n}=\mathbf{2 6}) \text { Fcia (\%) }\end{array}$ & ORP (IC 95\%) & $\mathbf{p}^{*}$ \\
\hline Complicationes post CPRE & $3(9)$ & $8(30.7)$ & $0.2(0.05-0.90)$ & 0.03 \\
Colongitis & $2(5.7)$ & $8(30.7)$ & $0.13(0.03-0.71)$ & 0.009 \\
\hline
\end{tabular}

IC 95\%: intervalo de confianza del 95\%; OR: odds ratio para la prevalencia.

${ }^{*}$ Calculada con la prueba de ji al cuadrado y considerando $p<0.05$ como significancia estadística.

(8/30; 7\%), con una OR de 0.2 (protectora para el diagnóstico a través de guía ASGE) calculada con la prueba de ji al cuadrado $(p=0.03)$ (Tabla 1).

\section{Mortalidad}

No se observó diferencia significativa entre ambos grupos.

\section{Día de la CPRE y días de estancia hospitalaria}

No se observó diferencia significativa entre ambos grupos.

\section{Día de la CPRE y colangitis}

Los pacientes diagnosticados a través de la guía ASGE presentaron menos días para la realización de la CPRE $(2.6 \pm 1.2 ; p=0.005)$ (Tabla 2).

\section{Discusión}

Las recomendaciones de la ASGE no son de amplio uso en nuestro medio, posiblemente por la falta de estudios que hayan evaluado el rendimiento de estos criterios.

De acuerdo con la guía propuesta por la ASGE, un paciente estratificado como de alto riesgo tiene más de un $50 \%$ de probabilidad de presentar coledocolitiasis ${ }^{4}$. En nuestro estudio, los pacientes estratificados como de alto riesgo tuvieron un $92.1 \%$ de probabilidad, lo que se encuentra muy por arriba de lo reportado en otros estudios, como el de Magalhaes, et al. ${ }^{8}$, que obtuvieron un $79.8 \%$.

El estudio de Benites, et al. ${ }^{9}$ halló una probabilidad del $88.4 \%$, pero el promedio de tiempo entre la sospecha diagnóstica y la realización de la CPRE fue de 10.8 días (en nuestro estudio el promedio fue de 2.6 días en el grupo ASGE), por lo que muchos pacientes cambiaron de estratificación de riesgo.
Tabla 2. Días de estancia intrahospitalaria y realización de CPRE en pacientes con coledocolitiasis diagnósticados a través de la guía ASGE en comparación con el método tradicional

\begin{tabular}{lccc}
\hline Variable & $\begin{array}{c}\text { Grupo } \\
\text { ASGE }\end{array}$ & $\begin{array}{c}\text { Grupo no } \\
\text { ASGE }\end{array}$ & $\mathbf{P}^{*}$ \\
\hline Dias de estancia intrahospitalaria & $7.3 \pm 2.9$ & $7.7 \pm 2.9$ & 0.3 \\
Dias de CPRE & $2.6 \pm 1.2$ & $5.2 \pm 1.9$ & 0.005 \\
\hline
\end{tabular}

*La diferencia de promedios se calculó con la prueba de t de Student y se consideró significancia estadística una $\mathrm{p}<0.05$.

Sethi, et al. ${ }^{10}$ obtuvieron, en el primer estudio prospectivo de la utilidad de la guía ASGE, una sensibilidad del $83.5 \%$, una especificidad $39.8 \%$ y un valor predictivo positivo del $75.6 \%$, con una probabilidad del $75.5 \%$, muy por debajo de lo que encontramos en nuestro estudio. Rubin, et al. ${ }^{11}$ realizaron un estudio para determinar la precisión del algoritmo propuesto para predecir la coledocolitiasis, y hallaron una probabilidad del $79 \%$; sin embargo, refieren que la sensibilidad y la especificidad son bajas para realizar procedimientos invasivos (CPRE). Adams y Hosmer $^{12}$ realizaron un estudio de cohorte retrospectivo y obtuvieron una probabilidad del $62.1 \%$, una especificidad del $73 \%$ y una sensibilidad del $46.7 \%$, así como una asociación independiente al contar con piedra vista por ultrasonido y unos valores de bilirrubina $>4 \mathrm{mg} / \mathrm{dl}$. Se concluye que, considerando la morbilidad y los costos asociados con los eventos adversos relacionados con la CPRE, el riesgo de resultados catastróficos ocasionales, la creciente disponibilidad de ultrasonido endoscópico y de resonancia magnética, y la creciente percepción médico-legal, la CPRE debe ser una intervención casi exclusivamente terapéutica. Recomiendan que una posible estrategia es el uso sistemático de ultrasonido endoscópico antes de la CPRE en caso de sospecha de coledocolitiasis sin signos de colangitis.

En nuestro estudio, en los pacientes sometidos al protocolo ASGE la CPRE se realizó más tempranamente que en los estudios que reportaron el día de la CPRE, pero esto adquiere más interés al analizar 
los días en que se realizó la CPRE y la aparición de colangitis, obteniendo una $p<0.5$, significativa a favor del grupo ASGE, lo que no se estudió en ninguno de los trabajos previamente mencionados. Tampoco realizaron una comparación de la presencia de colangitis intrahospitalaria, que fue más probable en el grupo no ASGE, con significancia estadística, por lo que fue un factor protector para colangitis someter al paciente al protocolo ASGE en nuestro hospital.

El mayor impacto de nuestra investigación lo encontramos al comparar la morbilidad de la CPRE en ambos grupos. Este método diagnóstico-terapéutico tiene una morbilidad global de hasta el $5 \%$, y es la razón por la que el resto de los estudios buscan encontrar elevados porcentajes de sensibilidad y especificidad para no abusar de ella. Sin embargo, en este estudio encontramos en el grupo no ASGE un porcentaje de morbilidad del $44 \%$, mientras que en el grupo ASGE fue del $9 \%$ (estadísticamente significativo; $p=0.03$ ), además de que en ningún otro estudio se ha comparado esta variable entre la guía propuesta por la ASGE y otro abordaje diagnóstico-terapéutico.

\section{Conclusiones}

En nuestro medio, el empleo de la guía ASGE para el diagnóstico oportuno de coledocolitiasis en pacientes con alto riesgo reduce la presencia de colangitis y las complicaciones tras la CPRE.

Sugerimos que este método sea empleado de manera sistemática por el servicio de cirugía general y endoscopia.

\section{Agradecimientos}

A todo el equipo quirúrgico del Hospital Regional del Instituto de Seguridad y Servicios Sociales de los Trabajadores del Estado, de Puebla.

\section{Financiamiento}

No se recibió ningún tipo de financiamiento para la realización de este estudio.

\section{Conflicto de intereses}

Los autores declaran no tener ningún conflicto de intereses.

\section{Responsabilidades éticas}

Protección de personas y animales. Los autores declaran que los procedimientos seguidos se conformaron a las normas éticas del comité de experimentación humana responsable y de acuerdo con la Asociación Médica Mundial y la Declaración de Helsinki.

Confidencialidad de los datos. Los autores declaran que han seguido los protocolos de su centro de trabajo sobre la publicación de datos de pacientes.

Derecho a la privacidad y consentimiento informado. Los autores han obtenido el consentimiento informado de los pacientes y/o sujetos referidos en el artículo. Este documento obra en poder del autor de correspondencia.

\section{Bibliografía}

1. Cervantes J, Rojas G. El mito de los cálculos inocentes. Cir Cir. 2001;69:92-6.

2. Singhvi G, Ampara R, Baum J, Gumaste V. ASGE guidelines result in cost-saving in the management of choledocholithiasis. Ann Gastroenterol. 2016;29:85-90.

3. Fitzgerald J, White M, Lobo D. Couvoisier's gallbladder: law or sign? World J Surg. 2009;33:886-9.

4. Maple J, Tamir B, Anderson M, Appalaneni V, Banerjee S, Cash BD, et al. The role of endoscopy in the evaluation of suspected choledocholithiasis. Gastrointest Endosc. 2010;71:1-9.

5. Hunt D. Common bile duct stones in non dilated bile ducts? An ultrasound study. Australas Radiol. 1996;40:221-2.

6. Urquhart P, Speer T, Gibson R. Challenging clinical paradigms of common bile duct diameter. Gastrointest Endosc. 2011;74:378-9.

7. Prat F, Meduri B, Ducot B, Chiche R, Salimbeni-Bartolini R, Pelletier G. Prediction of common bile duct stones by noninvasive tests. Ann Surg. 1999;229:362-8.

8. Magalhaes J, Rosa B, Cotter J. Endoscopic retrograde cholangiopancreatography for suspected choledocholithiasis: from guidelines to clinical practice. World J Gastrointest Endosc. 2015;16:128-4.

9. Benites G, Palacios F, Asencios J. Rendimiento de los criterios predictivos ASGE en el diagnóstico de coledocolitiasis en el Hospital Edgardo Rebagliati Martins. Rev Gastroenterol Perú. 2017;37:111-9.

10. Sethi S, Wang F, Korson A, Krishnan S, Berzin T, Chuttani R, et al. Prospective assessment of consensus criteria for evaluation of patients with suspected choledocholithiasis. Dig Endosc. 2016;28:75-82.

11. Rubin M, Thosani N, Tanikella R, Wolf D, Fallon M, Lukens F. Endoscopic retrograde cholangiopancreatography for suspected choledocholithiasis: testing the current guidelines. Dig Liver Dis. 2013;45:744-9.

12. Adams M, Hosmer A. Predicting the likelihood of a persistent bile duct stone in patients with suspected choledocholithiasis: accuracy of existing guidelines and the impact of laboratory trends. Gastrointest Endosc. 2015;82:88-93. 